Nicht editiertes Manuskript für einen Beitrag in einem Herausgeberwerk, wie folgt zitieren:

Schedler, Kuno: Forschungsannäherung an die managerialistische Verwaltungskultur. In: König, Klaus; Kropp, Sabine; Kuhlmann, Sabine; Reichard, Christoph; Sommermann, Karl Peter; Ziekow, Jan (Hrsg.): Grundmuster der Verwaltungskultur. Interdisziplinäre Diskurse über kulturelle Grundformen der öffentlichen Verwaltung. Baden-Baden: Nomos, 2014, S. 221-254

\title{
Forschungsannäherung an die managerialistische Verwaltungskultur
}

\author{
Kuno Schedler ${ }^{1}$
}

Verwaltungsforschung kann nicht dekontextualisiert erfolgen. Um sich selbst zu befähigen, Verwaltung empirisch erforschen zu können, müssen sich Forschende durch verschiedene Kontextschichten arbeiten, aus denen sich wichtige institutionelle Rahmenbedingungen für die Verwaltung und deren kulturelle Einbettung ableiten lassen. Nur so werden sie die relevanten Signale erkennen, die von den Mitgliedern des sozialen Systems Verwaltung ausgesandt werden. Der Beitrag definiert als Vorschlag vier solcher Schichten: Kulturbilder, Menschenbilder, Staatsbilder und Organisationsbilder, die in einem sozialen System vorherrschen. Der Anspruch eines tiefen Kontextverständnisses legt die Latte für vergleichende Verwaltungsforschung hoch. Gleichzeitig erklärt er, weshalb Public Management und Governance nur bedingt international verallgemeinerbare Erkenntnisse liefern können.

\section{Einleitung}

Seit den neunziger Jahren wird im deutschsprachigen Raum versucht, die öffentliche Verwaltung zu reformieren. Notwendig wurde dies, weil die alte, bürokratische Verwaltung nicht mehr in der Lage zu sein scheint, die aktuelle Dynamik ihrer Umwelt aufzunehmen und adäquat damit umzugehen. Europa als Ganzes, der deutschsprachige Raum eingeschlossen, blockiert sich selbst im Vergleich zur dynamischen Entwicklung globaler Konkurrenten wie China, Indien, Südamerika oder die USA. Politik und Verwaltung müssen ihre Agency, d.h. ihre Entscheidungs- und Handlungsfähigkeit, zurück gewinnen, wollen wir uns als globale Region nicht in die Unbedeutsamkeit abwerten lassen.

Ein wesentlicher Baustein einer solchen Veränderung ist die Stärkung der Steuerung im öffentlichen Bereich. Ein weiterer ist die Leistungsfähigkeit der öffentlichen Verwaltung, die den Ansprüchen eines schärfer werdenden internationalen Wettbewerbs nicht mehr genügt. Im vorliegenden Beitrag werfe ich einen vertieften Blick auf kulturelle Aspekte, die mit den Reformbemühungen interagieren.

Der Begriff der Kultur wird oft in Zusammenhang mit impliziten Verhaltensweisen, geteilten Werten und Erwartungen, Symbolen und/oder Umgang miteinander in Verbindung gebracht. Kultur ist damit oft als Gegenpol zu formalisierten Prozessen und Strukturen gedacht 
- was das Konzept selbst als Residualgrösse eher schwächt als stärkt. Im Kontext des vergleichenden Public Management schlägt Bouckaert (2007: 31 f.) vor, vier Stufen von Kultur zu unterscheiden:

a. Macro-Stufe: hier ist die Kultur einer Zivilisation angesprochen

b. Meso-Stufe: hier ist die Kultur einer Berufsgattung oder einer eines funktionalen Systems in der Gesellschaft angesprochen (professional culture / administrative culture)

c. Micro-Stufe: hier ist die Kultur einer spezifischen Organisation angesprochen

d. Nano-Stufe: hier ist die Kultur einer Teileinheit einer Organisation angesprochen

Der vorliegende Beitrag hat zum Ziel, eine ganzheitliche Herangehensweise zu beschreiben, wie Verwaltung in ihrem kulturellen Kontext verstanden werden kann. Er befasst sich primär mit den wechselseitigen, zirkulären Beeinflussungen der unterschiedlichen Stufen. Ich gehe davon aus, dass die Stufen auf unterschiedliche Weise miteinander verknüpft sind, d.h. sie stehen nicht isoliert für sich, sondern interagieren beständig. Dabei reproduzieren sie organisationale und gesellschaftliche Legitimation für die Verwaltung (DiMaggio und Powell 1983). Auf der organisationalen (Micro-) Stufe passt sich die öffentliche Verwaltung den Erwartungen bezüglich einer optimal strukturierten Organisation an, kann diese allerdings auch ein Stück weit selbst prägen (Meyer und Rowan 1977). Legitimation gegenüber der Gesellschaft entsteht beispielsweise über formalisierte Entscheidungsprozesse („,demokratische Legitimation“) oder über gute Qualität der Verwaltungsleistung (Schedler und Felix 2000). Im Zentrum unserer Betrachtung steht also beispielsweise nicht die gesellschaftliche Veränderung allein, die einen managerialistischen Zeitgeist enthalten kann, aber auch nicht eine spezifische Organisation im Sinne einer Case Study. Von Interesse ist für mich viel mehr der Übergang von Macro (via Meso) zu Micro und wiederum zurück zu Macro (vgl. auch Coleman 1990). Oder anders: welchen Einfluss hat der kulturell geprägte Kontext auf die Ausgestaltung der öffentlichen Verwaltung, und was bedeutet dies für die Art und Weise, wie die öffentliche Verwaltung mit ihren Stakeholdern interagiert?

\section{2. Öffentliches Management und Managerialismus}

Die öffentliche Verwaltung (nachfolgend nur noch "Verwaltung" genannt) im deutschsprachigen Raum hat ihr Gesicht seit dem ausgehenden letzten Jahrhundert massgeblich verändert. Prinzipien, die einst als Trutzburg gegen die Willkür feudaler Herrschaft eingeführt wurden, gelten heute in aller Regel als selbstverständlich: Legalität und Legitimität des Verwaltungshandelns sind wohl nicht das primär zu lösende Problem einer Reform im deutschsprachigen Raum. Daher wurden sie auch nicht vordringlich von Reformen wie beispielsweise der Wirkungsorientierten Verwaltungsführung thematisiert (Schedler 1995).

Seit den achtziger Jahren beherrscht ein neues Idealmodell der Verwaltung die Diskussion in den Verwaltungswissenschaften, aber auch in der Praxis: das am ökonomischen Denken gewachsene "New Public Management" (Aucoin 1990), in Deutschland als "Neues Steuerungsmodell" (Reichard 1994), in Österreich (Hammerschmid 2001) und der Schweiz (Buschor 1993; Schedler 1995) als "Wirkungsorientierte Verwaltungsführung" an die jeweiligen Verhältnisse adaptiert. Um es kurz zu fassen: Im Grunde geht es bei NPM, NSM und WoV um eine Umkehr der Steuerungslogik zwischen Politik und Verwaltung. Anstatt über Ressourcen und Regulierungen zu führen, wird der Fokus vermehrt auf die Leistungen und Wirkungen des Verwaltungshandelns gelegt. Damit soll erreicht werden, dass die Steuerung 
selbst effektiver wird. Für die Politik soll es eine Verwesentlichung ermöglichen, in der Verwaltung soll die Führung, das Management, gestärkt werden. Die Stärkung des Managements, verbunden mit den dazu notwendigen Führungs- und Steuerungsinstrumenten, bezeichnet man auch als "Managerialismus" (Pollitt 1990). Letztlich sind dies aber nur Zwischenziele auf dem Weg zu einer offenen, an den Bürgerinnen und Bürgern orientierten Verwaltung, die mit den beschränkten verfügbaren Ressourcen eine möglichst grosse gesellschaftliche Wirkung erzielt - creating public value nach Moore (1995).

Das ist an sich eine ausgesprochen positive Botschaft einer Reform, die allerdings in der praktischen Umsetzung teilweise auf weniger Goodwill als in der konzeptionierenden Literatur gestossen ist. Um weiter in die Thematik der managerialistischen Verwaltungskultur vorzudringen, muss zunächst der Begriff geklärt werden. Ich möchte die managerialistische Verwaltung so interpretieren, dass es sich um eine Verwaltung handelt, die über eine integrierende und bewusste Führung verfügt, der Handlungs- und Entscheidungsspielraum zugestanden wird, und die die Verantwortung für Gestaltung und Entwicklung des sozialen Systems "öffentliche Verwaltung" übernimmt. Hierbei halten sich die kommunikativen und sozialen Elemente die Waage mit den instrumentell-strukturellen. Mit dem Managerialismus, der nebst der neuen Institutionenökonomie als theoretische Grundlage des NPM gilt (Aucoin 1990), fliessen Erkenntnisse aus der Organisations- und der Managementforschung in die Verwaltungsreform ein. Ziel eines verbesserten Verwaltungsmanagements ist es, die Verwaltung als soziales System, als Organisation, effizienter und effektiver zu machen, als dies in der prä-managerialistischen Ära der Fall war. Die rein juristische Betrachtung und Praxis der Verwaltungssteuerung ist nach heutiger Auffassung dazu nicht in der Lage (Schindler 2010).

Der St. Galler Tradition (Rüegg-Stürm 2002) folgend, orientiere ich mich an einem Managementmodell, das die Verwaltung als Organisation versteht, die in ihrer Umwelt eingebettet ist und die sich beständig in kommunikativen Prozessen mit ihrer Umwelt auseinander setzt. Diese Umwelt lässt sich gedanklich in verschiedene Sphären unterteilen, wie beispielsweise die Wirtschaft, die Wissenschaft, die Technik oder die politisch-rechtliche Umwelt. Im Gegensatz zum privatwirtschaftlichen Unternehmen spielt jedoch eine weitere Umweltsphäre eine entscheidende Rolle, nämlich die Politik, die ihrerseits Recht definiert und vom Recht in ihrer Kontingenz beschränkt wird. Politik, Recht und Management bilden damit ein interdisziplinäres Dreiergespann, das sich gegenseitig beeinflusst und das je nach Situation in unterschiedlichen Bedeutungsgewichten aufeinander trifft (Brun et al. 2005). Dabei gilt es, im interdisziplinären Diskurs vorerst von einer Gleichberechtigung der Disziplinen auszugehen. Weder der Politikwissenschafter, noch der Jurist oder der Betriebswirt haben per se "recht", sondern es ist für jede Entscheidung neu abzuwägen, welche disziplinären Argumente jeweils wie viel Gewicht bekommen sollen. 
beauftragt; überwacht

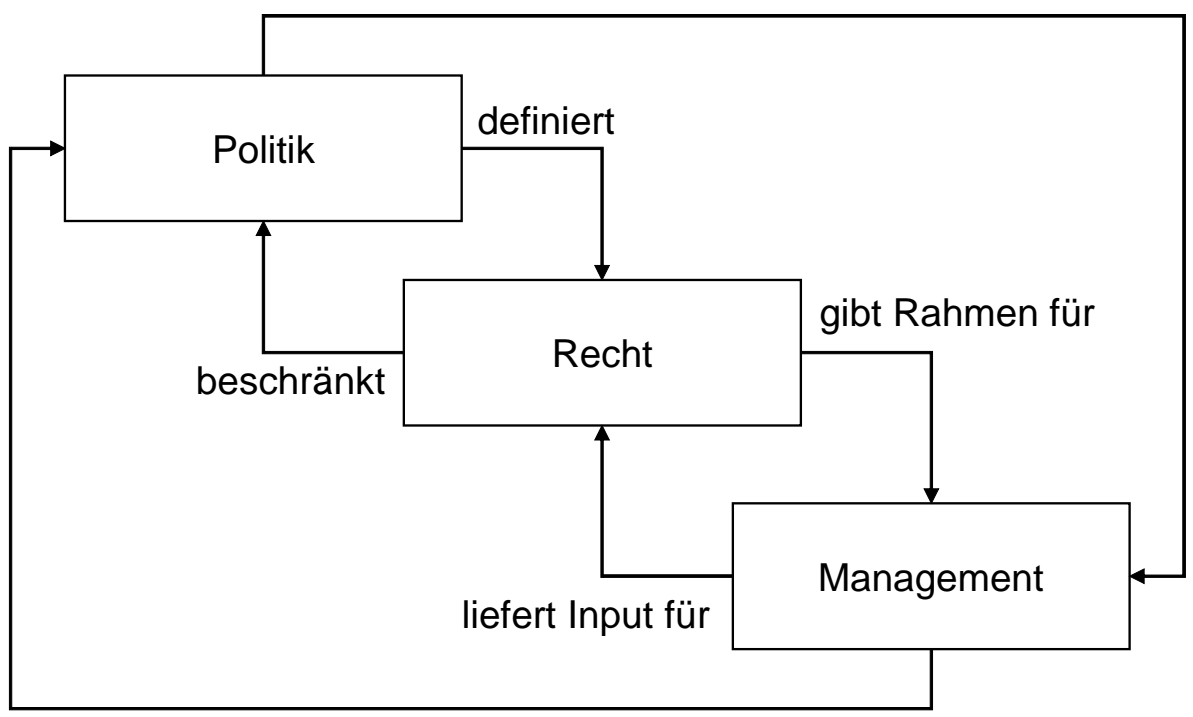

wird zum Thema für; beeinflusst

Abbildung 1: Verhältnis der Disziplinen (Brun et al. 2005)

Obwohl in der analytischen Betrachtung und in der Lösung von konkreten Praxisfällen von einer Gleichberechtigung der Disziplinen im Einzelfall ausgegangen wird, kann generalisierend gesagt werden, dass das Management in der Verwaltung an jene Spielräume gebunden ist, die ihm von der Politik oder vom Recht vorgegeben werden. Per Gesetz definiert damit die Politik den Entscheidungsfreiraum des Managements - der in aller Regel oft erheblich grösser ist, als dies die Führungskräfte in der Verwaltung wahrhaben wollen. Zusammenfassend kann also gesagt werden: Die managerialistische Verwaltung ist eine Verwaltung, der von Seiten der Politik bewusst ein grosser Management-Freiraum gewährt wird, und die dadurch gefordert wird, diesen Freiraum zu nutzen. Als Gegengeschäft für diesen zusätzlichen Freiraum stellt die Politik jedoch auch Erwartungen an die Verwaltung: mehr Effizienz (Kostenbewusstsein), mehr Effektivität (Leistungs- und Wirkungsbewusstsein), und gleichzeitig mehr Transparenz, um den bedrohlich wirkenden Kontrollverlust zu kompensieren. Die managerialisierte Verwaltung muss sich ihre neuen Freiheiten teuer erkaufen - und umso frustrierter reagiert sie, wenn sie für den bezahlten Preis der zusätzlichen Kontrolle nicht mit einer tatsächlichen Zunahme des Entscheidungsfreiraums entschädigt wird. Eine Vereinbarung über neue Steuerungsprozesse ist eine beidseitige Willensbekundung, die sowohl vom (politischen) Auftraggeber wie auch vom (ausführenden) Auftragnehmer eingehalten werden muss. Die Praxis zeigt, dass dem bei weitem nicht immer so ist. Daraus zu schliessen, dass ein stärker leistungsorientierter Ansatz in Deutschland nicht umsetzbar sei, wie dies einige Autoren (Bogumil 1997; Jann et al. 2004) behaupten, halte ich hingegen für fatalistisch und übermässig konservativ. Immerhin kann die Hoffnung auf Besserung zu Veränderungen in kleinen Schritten führen (Brunsson 2006).

Um die Veränderungen, die sich für eine Verwaltung aus der Tatsache ergeben, dass sie vermehrt "gemanagt" werden soll, überhaupt begreifen zu können, ist es notwendig, sich mit der Kultur der "traditionellen" Verwaltung in Westeuropa zu befassen. Nirgends ist Vaku- 
um, überall bestehen eingespielte Praktiken und routinisierte Verhaltensweisen (Giddens 1990), die von den Menschen als Strukturen erlebt und durch tägliche Wiederholung verfestigt werden. Die Strukturierung der öffentlichen Verwaltungen durch "Einschleifen" von bürokratischen Routinen ist vor New Public Management längst erfolgt, und jede Veränderung bedarf einer besonderen Begründung. Dies gilt nicht nur für die Verwaltung selbst, sondern auch für ihre Beobachter, die Verwaltungswissenschaftler.

Zwischen den Kulturen zeigen sich grosse Unterschiede, die in der Regel erst dann bewusst wahrnehmbar sind, wenn bewusst Vergleiche angestellt werden. Der französische Philosoph François Jullien nimmt beispielsweise China als kulturellen Kontrapunkt zum westlichen Denken, um seine europäische (bzw. französische) Wahrnehmung des Bestehenden zu erweitern. China fordert den Betrachter heraus, auch das Selbstverständliche in der eigenen Kultur zu hinterfragen.

"Das heisst, auf einem Umweg, ausgehend vom chinesischen Aussen, die impliziten, verborgenen Entscheidungen zu erhellen, die die europäische Vernunft getragen haben und die diese gerade deswegen nicht hinterfragen kann: denn es gibt das, was ich denke, aber auch das, von dem aus ich denke und das ich gerade deswegen nicht denke." (Jullien 2005: 15)

Kulturen sind demnach stets als Plural zu analysieren, als eine Vielzahl unterschiedlicher Alternativen des impliziten Umgangs mit Selbstverständlichkeiten. Verwaltung zu verstehen, bedeutet, sie als soziales System in ihrem lebendigen, historischen, aber auch zeitpunktbezogenen Kontext erfassen zu können. Vergleichende Verwaltungswissenschaft muss sich stets die Mühsal auferlegen, zunächst den Kontext eines Betrachtungsobjektes zu erlernen (Jann 1987). Ich wähle das Wort "erlernen" bewusst deswegen, weil die rein rationale Analyse für das Verständnis eines mit so viel Implizitem behafteten Feldes mit grosser Wahrscheinlichkeit zu kurz greifen wird.

Der Forscher kann demnach nicht direkt, mit seinem kulturell geprägten Fokus, die öffentliche Verwaltung anderer Systeme analysieren, ohne die mittransportierten Bilder zu berücksichtigen. Bilder sind immer interpretierbar, und die Assoziationen des Betrachters erfüllen sie mit geistigem Leben. Die Forscherin oder der Forscher (hier in Public Management) müssen die Bilder auf sich wirken lassen, sie interpretieren und - das verlangt die Wissenschaft kategorisieren, d.h. es muss ein Modell erstellt werden. Die möglichst breite Anwendbarkeit von Modellen verlangt es, dass sie die Wirklichkeit auf jene Zusammenhänge reduzieren, die dem Designer des Modells als relevant erscheinen. Dabei unterliegen wir oft der Versuchung, "den Westen" oder den deutschsprachigen Raum als eine kulturell homogene Einheit anzusehen, in der solche Modelle immer gleichartig funktionieren. Genau das Gegenteil ist der Fall, selbst innerhalb des deutschen Sprachraums, innerhalb eines Landes. Die relative Gleichheit der geschriebenen Sprache in Deutschland, Österreich und der Schweiz ist allzu verlockend, die Dinge zu vereinfachen und die Länder oder Regionen gleichzusetzen. Kontext ist aber nicht nur geschriebene Sprache, er ist viel komplexer, ist an Raum und Zeitpunkt gebunden.

Kulturbezogene Verwaltungsforschung ist nicht nur geeignet, um Verwaltungen in unterschiedlichen Ländern zu verstehen. Sie kann auch gute Dienste leisten, um Veränderungen der Verwaltung innerhalb eines mehr oder weniger konstanten Kulturkreises zu beobachten. 
Der Vergleich erfolgt dann nicht als Querschnitt, sondern im Längsschnitt, also über die Zeit. Streng genommen müsste zusätzlich ein Vergleich der unterschiedlichen disziplinären Sichtweisen auf die Verwaltung stattfinden, denn auch die Disziplinen in unterschiedlichen Kulturkreisen unterscheiden sich zum Teil fundamental. Auf diese zusätzliche Komplexitätsstufe möchte ich an dieser Stelle jedoch verzichten.

Wenn ich all das gesagt habe, so nehme ich nicht für mich als Person in Anspruch, diesen hohen Anforderungen gerecht zu werden. Vielmehr erwarte ich von der mir vertrauten Disziplin, den Verwaltungswissenschaften (mit Vertiefung im Public Management), dass sie als Forscherkollektiv in der Lage sein wird, diese Reise anzutreten und durch gemeinsame Erkenntnisanstrengung die Bilder so weit zu interpretieren, dass daraus ein neues gemeinsames Wissen, eine neue Wahrheit, entsteht. Die nachfolgenden Bilder(ge)schichten sollen also den wissenschaftlichen Diskurs anregen.

\section{Bilder(ge)schichten}

Den Begriff der Bilder habe ich ausgewählt, weil Bilder eine Materialisierung von Ideen sind, die es zu interpretieren gilt. Unabhängig davon, ob der Künstler oder die Künstlerin damit eine bestimmte Aussage machen wollte, löst ein Bild beim Betrachter oder der Betrachterin eine Assoziation aus. Mit der Betrachtung entsteht eine neue Wirklichkeit des Bildes, die dem Ursprung unter Umständen weit entfernt sein kann. Nun kann ich mich damit zufrieden geben, meine eigene Interpretation stehen zu lassen. Das ist für die Kunst legitim. Für die Forschung hingegen gilt es, die eigenen interpretativen Fähigkeiten so weit wie möglich zu schulen, so dass die eigene (konstruierte) Wirklichkeit jener Wirklichkeit möglichst nahe kommt, die die Menschen in der betrachteten Verwaltung erleben. Ich muss mich sozusagen diskursiv an das tiefere Verständnis einer Verwaltung annähern. Zentral ist dieses Heranarbeiten deswegen, weil mit den Bildern auch Erwartungen an das Handeln der Verwaltung entstehen, geprägt werden.

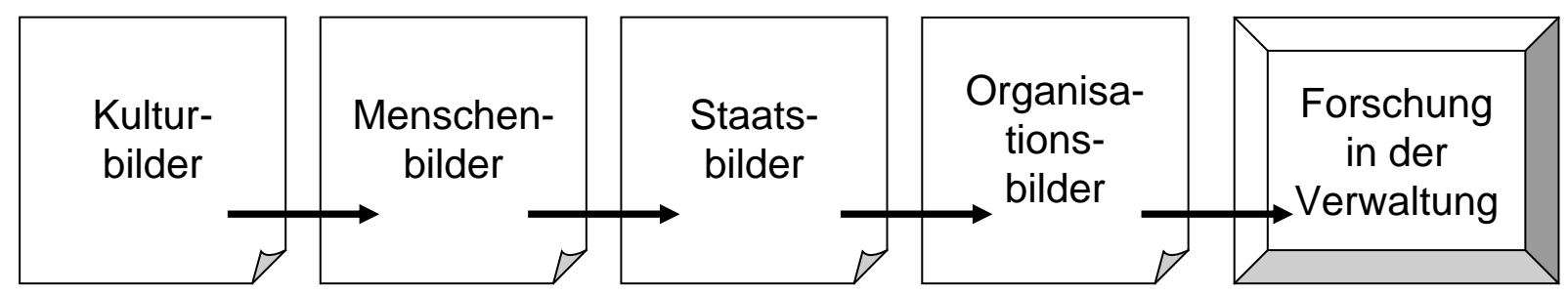

\section{Abbildung 2: Bilder(ge)schichten der Verwaltungsforschung}

Dabei durchschreite ich als Forscher bestimmte Schichten, Kontextwelten, die ich durchdringen muss, um überhaupt in der Lage zu sein, die jeweils nächstfolgende Schicht erfassen zu können (vgl. Abb. 2). Stark vereinfachend schlage ich vor, diese Schichten in einer Art gedanklicher Reise zu durchlaufen, indem die Kulturbilder, die Menschenbilder, die Staatsbilder und schliesslich die Organisationsbilder betrachtet werden müssen, die in einer Gesellschaft (oder einer anderen epistemischen Gemeinschaft) vorhanden sind. Erst danach kann ich in die Verwaltungsforschung eintreten, da ich erst mit diesem voraussetzungsreichen Rucksack an Vorwissen überhaupt erfassen kann, welche Diskurse in der Verwaltung statt- 
finden, welche Themen und unausgesprochene Wirklichkeiten das Verhalten der Menschen in der Verwaltung beeinflussen.

Lassen Sie mich wie versprochen versuchen, diese Schichten zu durchschreiten und jeweils mit Beispielen zu unterlegen, wie ich die Bilderanalyse verstehe. Wichtig ist mir dabei, dass ich die Unterschiede zwischen den Kulturen, zwischen den disziplinären Herangehensweisen oder den Praktiken der Verwaltung niemals wertend darstellen möchte.

\subsection{Kulturbilder}

Das Fundament unserer Analyse bilden die gesellschaftlichen Kulturbilder (Macro-Stufe der Kultur), die ein soziales System prägen. Sie sind daher die ersten, die wir erkennen müssen, um darauf aufbauend weitere Einsichten zu gewinnen. Jede Gesellschaft hat ihre geteilten Werte und Normen, die zu typischen Verhaltensweisen führen. Sie zu verstehen, für die Forschung erfassbar zu machen, ist ein ausgesprochen schwieriges Unterfangen. Man kann versuchen, die Werte der Menschen in einem Land zu erfragen, wie dies beispielsweise Hofstede (1991) macht. Kritisch ist die Frage, ob diese Aussagen die tatsächliche Kultur wirklich wiedergeben, aber immerhin verfügt die Forschergemeinschaft seit Hofstede (und ähnlichen Untersuchungen) über quantifizierbare Anhaltspunkte für kulturelle Unterschiede zwischen den Ländern.

Spätestens Schein (1992) hat in die Organisationsforschung die Erkenntnis eingebracht, dass Kultur (hier der Organisation, also die Micro-Stufe) nur in ihren für Forschende erkennbaren Manifestationen erfasst werden kann. Schein spricht von Artefakten, die dem Forscher oder der Forscherin Hinweise geben auf die gelebten Werte eines sozialen Systems, die wiederum in Grundprämissen verwurzelt sind, d.h. in nicht hinterfragten Glaubenssätzen über das Funktionieren der Welt und das Wesen des Menschen. Wenn wir also Kulturtypen definieren, so geschieht dies oft aufgrund von Artefakten, die wir als Forschende erkennen und typologisieren. Für staatliche Sozialprogramme in drei europäischen Ländern hat dies Jann (1983) in seiner Dissertation getan und dabei für Dänemark eine Kontaktkultur, für Grossbritannien eine Kontraktkultur und für Deutschland eine Regulierungskultur entdeckt. Auch wenn Jann selbst diese Ergebnisse immer wieder bescheiden relativiert, halte ich sie für ausgesprochen aufschlussreich, um den Verlauf von Verwaltungsreformen zu analysieren. Die Gleichheit der Rhetorik, die Kettl (1997) noch als weltweite Veränderung des Public Management interpretiert hat, führt nämlich in der Realität der Verwaltungsreform zu sehr unterschiedlichen praktischen Ausprägungen. Offensichtlich bestehen verschiedene Niveaus der Konvergenz von Verwaltungsreformen, die leicht zu Täuschungen führen (Pollitt 2002). Zum Teil hängt dies mit den drängenden Problemen zusammen, die mit der Reform gelöst werden sollen (Proeller und Schedler 2005), zu einem wesentlichen Teil aber auch mit dem Sensemaking (Weick 1995), das durch kulturelle Filter geprägt wird. Die normativen Werte des sozialen Systems fliessen Richtung gebend in die Verwaltungsreform ein (Wise 2002). Eine regelorientierte Verwaltungskultur, wie sie gemäss Jann in Deutschland vorherrscht, muss somit fast zwangsläufig zu einer stark regulierten und bürokratischen Variante des NPM führen. Politologen erklären dies unter anderem mit dem soziologischen Institutionalismus, wonach die Akteure in einem System nur jene Policies - darunter auch reform policies nach Barzelay und Gallego (2006) - wählen, von denen sie glauben, dass sie im bestehenden System Akzeptanz finden. 
Die Diskussion um Kultur - sei dies auf Gesellschaft oder Organisation bezogen - führt sehr schnell zu schillernden Ergebnissen. Kulturelle Erklärungen leisten gute Dienste, um etwa die Pünktlichkeit der Züge in der Schweiz, die Gründlichkeit der Bürokratie in Deutschland oder die Titelfreude der Offiziellen in Österreich zu verstehen. Oft sind aber Gründlichkeit, Unsicherheitsvermeidung oder blumige Ausformulierungen nur Ergebnisse einer tiefer sitzenden Quelle der Kulturen. Ohne Anspruch auf Vollständigkeit versuche ich in der Folge, vier mögliche Quellen für eine Gesellschaftskultur zu nennen und zu besprechen.

\subsubsection{Religion, Glaubenssätze}

Einer der wichtigsten kulturellen Faktoren in einer Gesellschaft sind tradierte Glaubenssätze, wie sie typischerweise in Religionen $\mathrm{zu}$ finden sind. Interessanterweise wird diese Thematik jedoch von der vergleichenden Verwaltungsforschung kaum aufgenommen, und es sind nur wenige wissenschaftliche Beiträge zu finden. Der Politologe Castles (1994) beschreibt beispielsweise, dass typische katholische Länder in Mittel- und Südeuropa in Politikfeldern, die stark durch Gender-Fragen bestimmt sind, deutlich erkennbare Unterschiede zu den eher protestantisch orientierten Ländern aufweisen. Zu Verwaltungsreformen schreibt er allerdings nichts. Viele Publikationen, die den Begriff der Religion explizit erwähnen, nennen sie schlicht als ein Differenzierungsmerkmal, wenn es um Gleichbehandlungsgrundsätze geht. $\mathrm{Ob}$ und wie die Religion auf die Ausgestaltung der öffentlichen Verwaltung einen Einfluss hat, ist bislang noch kaum systematisch untersucht worden.

Dennoch liegt der Zusammenhang intuitiv auf der Hand. Gerade die Rolle der Frau in der Gesellschaft, die Castles betont, wird eine direkte Auswirkung auf Kontingenzen für die Verwaltungsreform haben. Aber auch die Frage, welche Bedeutung etwa Verträge in einer Gesellschaft haben, ist relevant. Der chinesische Taoismus², der auch im heutigen China die führenden Kader beeinflusst, führt beispielsweise dazu, dass Verträge nicht als Fixgrösse in einer Geschäftsbeziehung angesehen werden, wie dies in Europa der Fall ist. Vielmehr betont der Taoismus, dass sich die Bedingungen des Lebens beständig verändern - mit der praktischen Konsequenz, dass auch die Verträge laufend angepasst werden müssen - so oft, dass sie in sich niemals den Stellenwert haben werden, den sie für uns haben. Dem NPM, das so viel Gewicht auf Leistungsverträge legt (Alford und O'Neil 1994; Boston 1995), wird dadurch ein wesentlicher Stützpfeiler entzogen. Zumindest ist zu erwarten, dass ein "NPM with chinese characteristics" entstehen müsste, wenn es in China umgesetzt werden soll - wofür es allerdings nur wenig Anzeichen gibt. Die Analyse der religiösen oder Glaubenswurzeln eines sozialen Systems führt zu wichtigen Hinweisen über die individuelle und kollektive Sinngebung, oder mit anderen Worten: sie ermöglicht eine Ahnung darüber, wie bestimmte Botschaften der Verwaltungsreform von den Mitgliedern der Verwaltung interpretiert werden, und welche Möglichkeiten sie für sich selbst sehen, auf diese Botschaften zu reagieren.

Lässt sich bezüglich des NPM (und damit der neu entstehenden managerialistischen Verwaltung) eine bestimmte religiöse Zuordnung oder Bevorzugung ausmachen? Der Ursprungsraum ist die Region des britischen Commonwealth (Neuseeland, Australien, Grossbritannien), sowie - was die Kontrahierung zwischen Parlament und Rat betrifft - die Niederlande,

2 Für China sind m.E. mehrere Quellen für Glaubenssätze zu nennen, allen voran der Taoismus, der Konfuzianismus und der Buddhismus. 
siehe das in Deutschland bekannt gewordene Tilburg Modell (Herweijer 1996). Beide Kulturräume sind nicht katholisch, sondern eher der protestantischen Ethik zuzuordnen. Diese ist tendenziell leistungsorientiert, arbeitsam, und eben accountable - und zwar im Hier und Jetzt und nicht erst in einer anderen transzendenten Welt. Ob dies der Grund für ein Modell ist, das sich ausgesprochen an Leistungen orientiert, das Leistungen kontrolliert und Verantwortlichkeiten ins Zentrum des Steuerungsmodells stellt, vermag ich nicht zu ergründen. Es wäre jedoch sicherlich einen vertieften Gedanken wert zu fragen, ob die klassischen katholischen Länder kulturell dem NPM weniger zugeneigt sind als die protestantischen.

\subsubsection{Sprache}

Die Sprache ermöglicht es, Dinge zu benennen. Im Sensemaking-Prozess nimmt die Benennung bestimmter Konzepte eine wichtige Rolle ein. Fehlt eine Bezeichnung für ein Konzept, so wird es ausgesprochen schwierig, darüber in einen Diskurs zu treten und in einem sozialen System eine gemeinsame Wirklichkeit zu entwickeln. Bouckaert (2007: 45) betont beispielsweise, dass es in vielen Sprachen keine Unterscheidung zwischen „policy“ und „politics“, zwischen „efficiency“ und „effectiveness“ oder keinen spezifischen Begriff für ",accountability" gibt. Nachdem dies jedoch Schlüsselkonzepte für das NPM nach neuseeländischem Muster sind, kann leicht nachvollzogen werden, dass das NPM in der Anwendung in nicht-englischsprachigen Ländern grosse Unterschiede aufweisen muss.

In einer Forschergruppe, die sich mit Reformen des öffentlichen Rechnungswesens befasst hat, wurde über den Begriff der Accountability diskutiert. Dabei wurde von den Teilnehmern aus verschiedenen europäischen Ländern erklärt, welchen Sinn dieser Begriff für sie hat. Seither führe ich diese Diskussion immer wieder mit meinen Studierenden und stelle fest, dass sich die Muster über die Jahre gleichen. Für die Deutschen ist die Übersetzung sprechend, die "Rechenschaftspflicht" ist eine rechtliche Verpflichtung, die entsprechend reguliert ist. Die Italiener sehen in der accountability eher ein paternalistisches Konzept, und ein Niederländer hat darin die Verantwortung des Menschen gegenüber Gott gesehen. Ich möchte diese Ergebnisse nicht überbewerten und halte sie eher für eine Anekdote, die aber wichtige Hinweise auf die Bedeutung der Sprache zu geben vermag.

Hinter Begriffen stehen kognitive Konzepte, die zu benennen es erst ermöglicht, dass epistemische Gemeinschaften entstehen. Hood (1998) verweist beispielsweise auf die Sprache des ökonomischen Rationalismus, die für das New Public Management typisch sei. Kettl (1997) stellt für Neuseeland fest, dass die Manager in der Verwaltung dort über ihr "Business" in einer Sprache sprechen, die sich nicht mehr von jener der privatwirtschaftlichen Manager unterscheidet. In der Literatur finden sich etliche Hinweise darauf, dass NPM eine Sprache mit sich gebracht hat, die man - gemäss dem Titel dieser Publikation - als managerialistisch bezeichnen könnte, was wohl nicht zuletzt auch dem Einfluss der grossen Beratungsfirmen auf die neuseeländische Verwaltungsreform zurück zu führen ist (Lapsley und Oldfield 2001). Der Längsschnitt zeigt vergleichend also eine Veränderung der Sprache in der Verwaltung, was wir als Artefakt für eine Veränderung der kognitiven Konzepte interpretieren könnten. Wer allerdings die Verwaltung besser kennt, weiss, dass diese Schlussfolgerung vermutlich nicht falsch, aber mit Vorsicht zu geniessen ist. In ihrem spezifischen Umfeld (Politik) hat die öffentliche Verwaltung gelernt, ihr Vokabular anzupassen, ohne zwingend auch die Tiefenprogrammierung zu verändern. Das kann auch beim NPM der Fall sein, wie Meyer und Hammerschmid (2006) für Österreich eindrucksvoll zeigen. 
Eine weitere sprachliche Herausforderung besteht in der Überbrückung professioneller Unterschiede in der Sprache. Internationale Erkenntnisse der Public Management Forschung werden nicht nur in Englisch, sondern auch mit einem speziellen wissenschaftlichen Code publiziert. Der Druck, in internationalen (anglo-amerikanisch geprägten) Journals zu publizieren, führt zu einer Übernahme des Forschungs- und Publikationsformats durch die Forschenden im deutschsprachigen Raum. Positiv daran ist, dass damit mehr scientific rigor entsteht, d.h. die Forschungsmethoden sind stringenter als noch vor zehn Jahren. Es geht aber gleichzeitig auch der Einfluss auf die Praxis verloren, da - zumindest in der Verwaltung vorläufig noch nicht zu sehen ist, dass Praktikerinnen und Praktiker "unsere" Journals lesen. Auch hier ist also eine Übersetzungsleistung gefordert, von wissenschaftlicher in praktische Formulierung und Darstellung. Wenn wir das nicht den Beratungsfirmen überlassen wollen, so müssen wir uns als Forschende auch hier engagieren.

\subsubsection{Macht}

Die Verteilung der Macht innerhalb eines sozialen Systems kann sowohl strukturell wie auch kulturell verstanden werden. Natürlich führen Strukturen zu einer Ordnung von Machtverhältnissen. Wer wofür verantwortlich ist, d.h. auch, wer für welchen Bereich entscheiden kann, zeigt sich in der Aufbaustruktur einer Organisation. Daneben gibt es aber auch die informelle Machtverteilung, die nicht formal strukturiert ist, die sich jedoch über die Einhaltung von sozial erwarteten Verhaltensweisen bestätigt. Eine der fünf Kulturdimensionen von Hofstede (1991) ist die vertikale Machtdistanz in einem sozialen System. Je höher die Machtdistanz, umso ausgeprägter wird die Hierarchie als Form der Governance gelebt. Es erstaunt den Europäer kaum zu hören, dass Frankreich in Europa der Leader im Ranking der Machtdistanz ist - weniger bekannt dürfte sein, dass die Schweiz eine vergleichsweise tiefe Machtdistanz aufweist, wobei hier zwischen den Sprachregionen recht erhebliche Unterschiede bestehen.

\begin{tabular}{|c|c|c|c|c|c|c|c|c|c|}
\hline $\mathrm{F}$ & $\mathrm{B}$ & $\mathrm{E}$ & $\mathrm{I}$ & $\mathrm{NL}$ & $\mathrm{D}$ & $\mathrm{UK}$ & $\mathrm{DK}$ & $\mathrm{A}$ & $\mathrm{CH}$ \\
\hline 15 & 20 & 31 & 34 & 40 & 42 & 42 & 51 & 53 & 58 \\
\hline
\end{tabular}

Tabelle 1: $\quad$ Rangierung europäischer Länder in Hofstede's Machtdistanz. (Hofstede 1991)

Interessanterweise lassen sich für Europa keine statistischen Zusammenhänge zwischen der Machtdistanz und dem Umsetzungsgrad von NPM herstellen. Vielmehr scheint mir die Frage, wie die Veränderungsprozesse ablaufen, von der Machtdistanz und allgemein von der Machtverteilung abhängig zu sein. Eine dezentrale, partizipative Reform dürfte für Frankreich ein kulturell fast unmögliches Unterfangen sein. Kuhlmann (2006) zeigt beispielsweise für Frankreich, dass die dortigen Reformen auf der lokalen Ebene kaum zu Verschiebungen des tatsächlichen Machtgefüges geführt haben - und wohl auch nicht führen werden. Demgegenüber ist zu erwarten, dass eine zentral geführte, hierarchisch organisierte Reform in der Schweiz zum Scheitern verurteilt wäre

Etwas allgemeiner formuliert, hat auch die horizontale Machtverteilung in einem sozialen System eine grosse Auswirkung auf seine Zusammenarbeitsformen. Für den öffentlichen Raum bedeutet es, dass die Möglichkeiten, Spielvarianten der Public Governance anzuwenden, durch die existierende Machtverteilung mit beeinflusst werden. Wenn beispielsweise eine gesellschaftliche Elite besteht, die sich relativ mobil zwischen den verschiedenen Syste- 
men Politik, Verwaltung und (staatlich dominierte) Wirtschaft hin und her bewegt wie in Frankreich, so ist die Kontingenz für die Public Governance hoch: durch die ohnehin vorhandene personelle Vernetzung der Sektoren sind die Möglichkeiten des so genannten Privatsektors, mit dem öffentlichen Sektor partnerschaftliche Beziehungen (Public-Private Partnerships) einzugehen, viel unkomplizierter zu eröffnen als in einem System mit rigoroser Trennung. Die von Kettl (1993) thematisierte neue Macht(ver)teilung zwischen public und private wird durch die faktische informelle Vernetzung der Abgänger der Grands Ecoles erheblich gelindert - ja, es stellt sich sogar die Frage, ob für viele Länder Public Private Partnerships faktisch überhaupt eine neue Erscheinung sind.

In einem Land wie China, das sich - diesmal in konfuzianischer Tradition - über seine ganze Geschichte hinweg nach Einigung gesehnt hat, bekommt Macht eine weitere Bedeutung: sie wird als das legitime Mittel angesehen, um das Riesenreich zusammen zu halten. Governance-Formen, die diese Aufgabe schwächen, sind ausserhalb des Möglichen. Die Hierarchie ist offensichtlich passend, am anderen Ende der Skala möglicher Governance-Formen steht hingegen der Markt, der in China längst Einzug gehalten hat. Selbst dieser wird allerdings so organisiert, dass er seine positive Wirkung grösserer Effizienz erzielen kann, im Grunde aber den Einfluss des Machtapparates nicht unterwandert. Ein spannendes Beispiel ist die Situation im Handy-Markt. Um das Monopol des damals einzigen staatlichen Anbieters zu durchbrechen, wurde der Chinesische Markt in zwei Teile aufgeteilt, einen nördlichen und einen südlichen. Die daraus entstehenden zwei Firmen wurden aufgefordert, sich von nun an wettbewerblich zu bekämpfen. Später kam eine dritte Firma dazu, so dass heute drei staatliche Mobile Phone Companies zueinander im Wettbewerb stehen. Statt Public-Private Partnerships gibt es also Public-Public Competition, und zwar nicht nur im Telefoniebereich, sondern auch bei den Fluggesellschaften, den Stahlwerken und vielen anderen Bereichen. Stabilisierende Macht als Kulturgut.

\subsubsection{Kollektives Gedächtnis}

Das vierte und letzte hier erwähnte Kulturphänomen ist das kollektive Gedächtnis eines sozialen Systems. Auch dieses definiert Kontingenz. In unserem Zusammenhang ist vor allem Politik von Interesse, und zwar im weiten Sinne der Policies (also: was darf überhaupt getan werden), der Politics (wie darf Politik gemacht werden) sowie der Polity (welche politischen Strukturen dürfen institutionalisiert werden). Das kollektive Gedächtnis äussert sich als Kulturphänomen beispielsweise über Tabus und Reflexe, aber auch über Erwartungen an Abläufe, die historisch bestätigt scheinen. Daraus ergeben sich Pfadabhängigkeiten, die die Spannweite möglicher Entscheidungen im Einzelfall deutlich einschränken (Pierson 2000).

Zum kollektiven Gedächtnis zähle ich tatsächliche einschneidende Ereignisse, wie etwa für die Schweiz die Schlacht von Marignano (1515), die nebst anderen brutalen Schlachten in Italien dazu geführt hat, dass sich die Schweiz aus dem Söldnerwesen verabschiedet und seine damals europaweit beachtliche militärische Stärke aufgegeben hat. Das heute hartnäckige Festhalten der Schweizer an der Neutralität wird also im 16. Jahrhundert begründet, bestätigt durch den Vertrag von Versailles, und genährt durch das erfolgreiche "neutrale" Überstehen der beiden Kriege des 20. Jahrhunderts. Das Beispiel zeigt auch, dass beim kollektiven Gedächtnis neben die historisch belegten Ereignisse immer auch ein Stück Mythos tritt, und dass ein soziales System diese beiden Bereiche kaum zu trennen vermag. Geschichte wird stets aus der Perspektive der Gegenwart geschrieben und interpretiert. Auch sie ist 
letztlich ein Konstrukt. Wie dem auch sei: die Neutralität aufzugeben, wäre für die Schweiz ein Tabubruch, den kein Politiker überstehen kann. Diese Option liegt ausserhalb der Kontingenz des politisch Denkbaren.

Solche Tabus und Reflexe kennt jedes soziale System, und es ist Aufgabe des Forschers oder der Forscherin, sie in sein oder ihr Bewusstsein zu bringen, wenn später die Verwaltung analysiert werden soll. Für die Erforschung der managerialistischen Verwaltung stellen sich Fragen wie: Gibt es Tabus, die durch die Reform verletzt werden? Lösen einzelne Elemente der Reform Reflexe des Systems oder seiner Mitglieder aus, die zu Folgen führen? Werden durch bestimmte Themen Erwartungen geweckt, die im negativen Fall zu Abwehrreaktionen führen, oder die im positiven Fall das soziale System zu mobilisieren vermögen?

\begin{tabular}{|l|l|l|l|l|}
\hline $\begin{array}{l}\text { Quellen der } \\
\text { Gesellschafts- } \\
\text { kultur }\end{array}$ & $\begin{array}{l}\text { Religion, Glau- } \\
\text { benssätze }\end{array}$ & Sprache & Macht & $\begin{array}{l}\text { Kollektives Ge- } \\
\text { dächtnis }\end{array}$ \\
\hline $\begin{array}{l}\text { Wirkung auf } \\
\text { die Gesellschaft }\end{array}$ & $\begin{array}{l}\text { Wertvorstellun- } \\
\text { gen }\end{array}$ & $\begin{array}{l}\text { Kognitive Kon- } \\
\text { zepte }\end{array}$ & $\begin{array}{l}\text { Zusammenar- } \\
\text { beitsformen }\end{array}$ & $\begin{array}{l}\text { Tabus, Reflexe, } \\
\text { Erwartungen }\end{array}$ \\
\hline Kontigenzen & $\begin{array}{l}\text { Individuelle } \\
\text { und kollektive } \\
\text { Sinngebung }\end{array}$ & $\begin{array}{l}\text { Epistemische } \\
\text { Gemeinschaften }\end{array}$ & $\begin{array}{l}\text { Governance } \\
\text { Möglichkeiten }\end{array}$ & $\begin{array}{l}\text { Möglichkeiten } \\
\text { für Policies }\end{array}$ \\
\hline
\end{tabular}

Tabelle 2: Kulturphänomene und ihre Auswirkungen

Zusammenfassend zeigt die Tabelle 2 auf, welche Quellen der Gesellschaftskultur ich aus momentaner Sicht für besonders relevant erachte. Gleichzeitig versuche ich darzustellen, über welche Transmissionsgrössen ("Wirkung auf die Gesellschaft") die Kulturphänomene letztlich die möglichen Ausprägungsformen einer öffentlichen Verwaltung einschränken. Jede Kultur erlaubt nur bestimmte Formen von öffentlicher Verwaltung.

\subsection{Menschenbilder}

Aus den in einem sozialen System dominierenden Kulturbildern ergeben sich Vorstellungen über das Wesen des Menschen an sich, sowie als Akteur in spezifischen Umfeldbedingungen. Wer sich mit Menschenbildern befasst, wird verschiedene Ansätze der Forschung und Strukturierung vorfinden. Die für uns wichtigsten möchte ich in der Folge kurz ansprechen.

\subsubsection{Menschenbilder in der Organisationsforschung}

Aus der Organisationsforschung kennen wir die Entwicklung der Menschenbilder vom homo oeconomicus über den social man, den self-actualizing man zum complex man (Wunderer 1997). Der homo oeconomicus ist geprägt durch den Willen, seinen individuellen Nutzen zu maximieren. Er reagiert auf Anreize von aussen, kann also über die Gestaltung der pekuniären Strukturen (z.B. Leistungslohn) zu einem bestimmten Verhalten gebracht werden. Dieses Menschenbild liegt den traditionellen ökonomischen Theorien zugrunde, die ihrerseits wiederum als eine Quelle des New Public Management gelten (Grüning 2000). Neuere Forschungen zeigen allerdings, dass diese Annahme, die für die Ökonomik für lange Zeit eine Art unhinterfragter Glaubenssatz zu sein schien, nur bedingt zutrifft. Experimente der neueren Ökonomie zeigen, dass Individuen in bestimmten Situationen die Fairness über 
die eigene kurzfristige Nutzenmaximierung stellen. Fehr und Gächter konnten zum Beispiel zeigen, dass ihre Probanden in einer spieltheoretisch simulierten Situation persönliche Nachteile auf sich nahmen, um unfaire Spieler zu bestrafen. Sie nennen dies "altruistische Bestrafung" (Fehr und Gächter 2002). Auch Ergebnisse der neu entstehenden Disziplin der Neuroökonomie machen deutlich, dass die Idee des homo oeconomicus nicht aufgegeben, aber differenziert werden muss. So konnte beispielsweise nachgewiesen werden, dass Angebote, die als unfair empfunden werden, andere Hirnaktivitäten auslösen als faire Angebote (Helmuth 2009). Spannend ist ausserdem, dass dieselben "unfairen" Angebote weniger Emotionen auslösen, wenn sie vom Computer kommen, als wenn sie von einem Menschen gemacht werden. Die Rationalität der Entscheidung erfährt dadurch eine intersubjektive Komponente.

Der homo oeconomicus hat für die managerialistische Verwaltung eine tragende Rolle als Menschenbild, denn er steht für Leistungslohn und Anreizsysteme. Ob diese Systeme hingegen die Verwaltung nachhaltig verändern werden, ist kritisch zu betrachten. Neben ihm zeitlich allerdings mit leicht späterem Auftreten - steht das Bild des homo socialis, der den Wunsch verfolgt, in ein soziales Umfeld eingebettet zu sein. Er reagiert auf soziale Normen, Akzeptanz, Kommunikation. Dies würde die Abweichungen bei den Probanden von Fehr und Gächter (2002) erklären, die sich altruistisch verhalten, um die sozialen Normen zu schützen. Der neueren Organisationstheorie liegt ein sozialeres Menschenbild zugrunde. Weick's Sensemaking ist sowohl ein individueller wie auch ein sozialer Prozess (Weick 1995). Die Kommunikation zwischen den Individuen spielt dabei eine wesentliche Rolle.

\subsubsection{Leitbild-abhängige Menschen- und Rollenbilder}

Für die Verwaltungsreform relevant sind auch Menschenbilder, die den Mitarbeitenden in der Verwaltung bestimmte Rollenerwartungen zuweisen. Im Bürokratiemodell der Verwaltung (Weber 1947), das im Wesentlichen ein Maschinenmodell der Organisation ist, sind die Menschen Teil der Maschine und haben sich regelkonform zu verhalten. Dieses - durchaus nicht negativ konnotierte - Bild dürfte der traditionellen Rechtswissenschaft zugrunde liegen. Abweichungen von der generell-abstrakten Regel werden als Willkür sanktioniert. Ein guter Beamter ist, wer sich an die Regeln hält, denn dann funktioniert die Maschine und produziert keine (menschlichen) Fehler. Auftretende Probleme werden durch Perfektionierung des Regelwerks gelöst. Hier wird offensichtlich, dass die Menschenbilder und die später zu beschreibenden Organisationsbilder eng miteinander verbunden sind.

Politikwissenschaftliche und soziologische Studien zeigen, dass die Verwaltung nicht nur maschinell ausführender Arm der Politik ist, sondern dass sie einen wesentlichen Beitrag zur Formulierung und Umsetzung von Politiken leistet (Kenis und Schneider 1991; Mayntz 1997). Mit einem Rollenbild, das den Menschen als rein ausführenden Teil der Maschine Verwaltung definiert, lässt sich hier kein Staat machen. Vielmehr versteht diese Sichtweise den Mitarbeiter in der Verwaltung als politischen Menschen, der in der Lage sein muss, politische Vorgaben in seine tägliche Arbeit zu übertragen und umgekehrt. Public Manager tragen damit die Verantwortung für die Übersetzung zwischen zwei Rationalitäten: hier die politische Rationalität des demokratisch übergeordneten politischen Systems, da die Rationalität des fachlich überlegenen Verwaltungssystems (Schedler und Proeller 2000). 
$\mathrm{Zu}$ dieser Rolle fügt die managerialistische Verwaltung ausserdem jene des Entrepreneurs, der seine Aufgabe mit mehr Ausführungsfreiheit und -verantwortung erfüllen soll (Osborne und Gaebler 1992; Hood 1998). Die strukturelle Massnahme, die dieses Menschenbild fordert, ist das Globalbudget (Buschor 1993) - in Englisch "one-line budget" (Hood 1991) oder "lump-sum budget" (Thompson und Jones 1986). Dieses Menschenbild geht von einem verantwortlich handelnden, am Gemeinwohl orientierten, aber auch Effizienz und Effektivität fördernden Mitarbeiter aus. Die Verallgemeinerung ist allerdings nur zum Teil richtig, wie Helmuth (2010) gezeigt hat. Ausserdem verändern sich Menschenbilder im Verlauf der Zeit. Kolbe (2009: 163) zeigt beispielsweise auf, wie sich das Beamtenbild durch die US amerikanische National Performance Review verändert hat: Von „Bürokraten halten sich an Regeln. Diese werden auch nicht bei positiven Effekten einer Regelabweichung überschritten. " $\mathrm{zu}$ : „Bürokraten brechen Regeln, wenn es der daraus resultierende Nutzen rechtfertigt. Das Verhalten ist jedoch weiterhin in Institutionen eingebettet."

\subsubsection{Kulturell geprägte Menschenbilder}

Neben diese kontextbefreiten theoretischen Ansätze sind zweifellos jene Menschenbilder zu stellen, die sich aus einer bestimmten Kultur ergeben. Dies ist die Logik des hier vorgestellten methodischen Vorgehens. Es ist die Frage zu stellen, wie ein soziales System seine eigenen Mitglieder wahrnimmt, welche Erwartungen es an die Verhaltensweisen der Menschen im System hat. Dazu gehört eine Reihe von Themen, die insgesamt zu einer komplexen Gesamtheit zusammenfinden.

Ein herausragendes Beispiel ist etwa der Wert, der dem Individuum zugemessen wird. In der konfuzianischen Tradition Chinas bestimmt sich der Wert des Einzelnen über die Zugehörigkeit zur Gruppe, zunächst und primär zur Familie. Demgegenüber steht das Menschenbild etwa der meisten Amerikaner, die dem Individuum losgelöst von sozialen Bindungen eine hohe Werthaftigkeit zumessen. Die persönliche Freiheit steht über allem.

Wollen wir die managerialistische Verwaltung in ihrem Kontext erfassen, so sind wir gefordert, das kulturell geprägte aktuelle Menschenbild des umgebenden sozialen Systems kennen zu lernen. Wer ist der Einzelne, wer ist er in seiner Gruppe, wer ist er im Verhältnis zur Gemeinschaft, zum Staat? Wenn der Einzelne keine Bedeutung für den Staat hat, so macht es wenig Sinn, in der Verwaltung Kundenorientierung zu propagieren. Diese Forderung muss ins Leere stossen, oder aber sie wird absurd und führt zu fehlgeleiteten Reaktionen. Als Beispiel mag das Verhältnis von NPM und Korruption dienen. Die Diskussion darüber, ob NPM zu Korruption führt oder nicht, kann u.a. vor diesem Hintergrund interpretiert werden: wo Korruption an der Tagesordnung ist, wirkt die Forderung nach Kundenorientierung fast schon zynisch (Anechiarico 1998). Dass die Kundenorientierung jedoch die Korruption fördern würde, wie dies auch schon befürchtet wurde, konnte bislang nicht bestätigt werden (Von Maravic und Reichard 2003).

\subsection{Staatsbilder}

Das dritte Element, das es auf dem Weg zu einer kontextbezogenen Verwaltungsforschung zu erfassen gilt, sind die Staatsbilder, die einem sozialen System eigen sind. Nachdem wir geklärt haben, welche Kulturbilder einer Gesellschaft eigen sind, und nachdem wir vertie- 
fend die Menschenbilder untersucht haben, die vorherrschen, sind wir gut vorbereitet, uns mit den dominierenden Staatsbildern zu befassen. Die Staatsbilder entstehen nämlich unter anderem als Reaktion auf die ersten beiden Schichten - der Staat muss sich in Kultur und Menschen einbetten, kann nur in diesem Rahmen existieren. Wichtige Fragen sind: Wer ist der Staat? Welche Aufgaben hat der Staat zu erfüllen? Wie ist das Verhältnis des Staates zur Wirtschaft? Auch hier lassen sich im Quervergleich grosse Unterschiede ausmachen. Was beispielsweise Pollitt und Bouckaert (2000) als administrative culture bezeichnen, würde ich in die Kategorie der Staatsbilder einordnen, nämlich die Unterscheidung von Rechtsstaat und public interest, also ein Staat, der seine Massnahmen nicht primär an den rechtlichen Vorgaben ausrichtet, sondern am öffentlichen Interesse. Natürlich ist mit den beiden Autoren übereinzustimmen, dass dies auch Auswirkungen auf die Verwaltungskultur haben wird, jedoch scheint mir die Unterscheidung deutlich grundsätzlicher zu sein als "nur" die Kultur der Verwaltung. Es ist eine völlig andere Staatsidee.

Ein Quervergleich macht intuitiv deutlich, welche grossen Unterschiede zwischen den Staatsbildern allein in Europa bestehen. Der Sozialstaat nach Skandinavischer Prägung greift (mit seiner Verwaltung) viel mehr in das Leben der Bürgerinnen und Bürger ein als der britische Staat, der auf Eigenverantwortung und Wettbewerb basiert. Im Etatismus, der beispielsweise in Frankreich vorzufinden ist, soll der Staat die Probleme der Bürger lösen, kann sich aber über seine elitären Institutionen zu einem eigenständigen, schwer zu kontrollierenden Moloch entwickeln. Dem gegenüber steht das in der Schweiz vorherrschende Bild, dass der Staat grundsätzlich nur subsidiär wirken soll, d.h. er hat sich aus allem heraus zu halten, was die Bürgerinnen und Bürger selbst erledigen können. Die hartnäckige Verweigerung einer Professionalisierung staatlicher Strukturen (z.B. die Parlamente) ist ein sprechendes Beispiel für dieses Staatsbild: wer seinen Job für den Staat gemacht hat, soll wieder zurück ins Glied, damit er keine Macht ansammeln kann (Schedler 2008).

Im Längsschnitt, also in Bezug auf die zeitliche Entwicklung von unterschiedlichen Staatsideen, ist zeitgleich mit dem NPM auch der Wandel vom Leistungs- zum Gewährleistungsstaat erfolgt (Mastronardi und Schedler 1998; Mastronardi 2000; Hoffmann-Riem 2001). Noch bis in die 90er Jahre war der Begriff des Gewährleistungsstaates im deutschsprachigen Raum unbekannt, obwohl die Auslagerung von Leistungen im öffentlichen Interesse durchaus eine lange Tradition hat. Gerade in der Schweiz, die schon immer über eine ausgeprägt starke Zivilgesellschaft verfügte, ist dies nicht neu. Allerdings hat sich die Form der Auslagerung verändert, jedenfalls im wohlfahrtsstaatlichen Bereich: von der Subventionierung zur Leistungsvereinbarung (Ruflin 2006; Zauner et al. 2006). Dies verändert nicht nur die Beziehungen zwischen Organisationen, sondern auch die Kultur innerhalb der Organisationen.

Der Gewährleistungsstaat charakterisiert sich dadurch, dass öffentliche Aufgaben nicht zwingend durch den Staat bzw. die Verwaltung selbst erfüllt werden müssen, sondern dass sie auch an Dritte delegiert werden können. Die "Leistungstiefe" (Naschold et al. 1996) verändert sich also. Daraus entstehen die im Moment diskutierten Erfüllungsformen des Contracting Out, der Public-Private Partnerships, aber auch eine ganze Fülle von Spielformen des Zusammenwirkens von Privaten (inkl. Non-Profit Organisationen) mit dem Staat. Dies ist eine neue, für Viele unbekannte Form von Staatsbild: es ist nicht mehr nur gut, was der Staat macht, sondern auch, wofür er weiterhin verantwortlich bleibt, es aber nicht selbst tut. Daraus ergeben sich aber auch eine ganze Anzahl neuer Probleme, die vereinfachend als "clash of cultures" zwischen privatwirtschaftlich-ökonomisch orientierten und staatlich- 
gemeinwohlorientierten Organisationen bezeichnet werden können. Das Gewinnstreben der Privaten ist den Beamten suspekt, während die z.T. schwerfälligen Entscheidungswege im staatlichen Bereich von den Privaten kaum verstanden werden. Für den wohlfahrtsstaatlichen Bereich zeigt Ruflin (2006) ausserdem, dass die Einführung von Leistungsvereinbarungen in der Schweiz auch eine Machtverschiebung mit sich brachte: die Leistungsdefinition erfolgt jetzt nicht mehr bei den Wohlfahrtsorganisationen (NPOs), sondern in der Auftrag gebenden Verwaltung.

Die Staatsbilder können sich m.E. nur in Abhängigkeit von den Menschenbildern entwickeln, die in einem sozialen System vorherrschen. In einem Klima, in dem rein individualistische Nutzenmaximierung erwartet wird, muss der Staat eine andere Funktion wahrnehmen, als in einem Klima sozial verantwortlicher und engagierter Bürgerinnen und Bürger. Der Entwicklungsgrad der Zivilgesellschaft mag dafür ein Indikator sein, denn gerade die zivilgesellschaftlichen Organisationen sind Beispiel dafür, dass sich die Bürgerinnen und Bürger um das Wohlergehen des Gesamten (inkl. die Umwelten) kümmern.

\subsection{Organisationsbilder}

Schliesslich gilt es, sich in einem vierten Element über die Bilder Gedanken zu machen, die ein soziales System über das Wesen und die Rolle der Organisation entwickelt. Was ist Zweck und Aufgabe von Organisationen? Welche Beziehung hat die Organisation zu anderen sozialen Subsystemen? Wie steht das Individuum zur Organisation? Morgan zeigt eindrücklich, dass unsere Analysen und Erklärungen von Organisationen oft mit Metaphern verbunden sind, „, die es uns ermöglichen, Organisationen differenziert und doch nur ausschnittweise zu betrachten und zu begreifen" (Morgan 2002: 15). Da sich Metaphern stets auf bereits Bekanntes beziehen und eine Beziehung dazu herstellen, sind sie selbst kulturell geprägt. Zu analysieren, mit welchen Metaphern Organisationen in einem Kulturkreis beschrieben werden, heisst demnach, sich auf das Referenzsystem dieses Kulturkreises einzulassen. Wird die Organisation als Familie bezeichnet, so ist zu klären, welche Vorstellung von Familie hinter dieser Metapher steckt: ist es vor allem ökonomische Loyalität? Ist es emotionale Verbundenheit? Ist es gar eine Last?

Auch hier zeigt sich, wie stark dieses Element der Analyse mit den anderen verknüpft ist. Würde man sich tatsächlich sequenziell analytisch durch die Schichten arbeiten, so entstünden zunehmend Redundanzen, da viele Fragestellungen zu Interrelationen zwischen den Schichten mal aus dieser, mal aus der anderen Sicht angegangen werden. Ich meine aber, dass in der Sozialforschung Redundanzen notwendig sind, da sie die Erfassung der Komplexität sozialer Prozesse erst ermöglichen. Auch die Forschung soll wirtschaftlich sein, soll effizient organisiert werden. Aber sie muss auch umfassend, Komplexität miteinbeziehend und vor allem problemorientiert erfolgen.

Organisationsbilder können erheblich variieren, je nach Land, Zeitpunkt, oder auch je nach professioneller Sichtweise. Ist die Organisation deswegen existent, weil sie die geringsten Transaktionskosten für die Mitglieder der Organisation erlaubt? Oder wird sie als Ort gesehen, wo Gleichgesinnte (im eigentlichen Sinn des Wortes: Menschen, die in der Organisation den gleichen Sinn verfolgen) gemeinsam ein Ziel verfolgen? Liegt dieser Sinn primär im Output der Organisation, oder geht es den Mitgliedern um deren Wirkung? Oder aber: kann 
es auch der Sinn der Organisation sein, dass sie ihre Mitglieder sozial verbindet, wie dies bei Vereinen der Fall ist?

Die Organisationsbilder können eine sehr grosse Wirkung auf die Art und Weise haben, wie die Organisation funktioniert. Ein Beispiel ist die Gestaltung einer modernen Pflege von Menschen. Mosher und Burti (1992) propagieren etwa eine Psychiatrie, die nicht institutionell strukturiert ist und auf Medikamenten basiert, sondern die auf die soziale Einbindung der Nutzer von gemeindepsychiatrischen Zentren baut:

"Die fortlaufende Veränderung in der Organisation des Versorgungssystems in Italien hat einen allmählichen, aber deutlich beobachtbaren Wandel in der Art der Wünsche bewirkt, die von Patienten und Angehörigen geäussert werden. Wünsche werden differenzierter geäussert. Das ist eine Funktion der Dezentralisierung von Diensten und besonders der Qualität der angebotenen Behandlungen ... In der Öffentlichkeit ändern sich die Einstellungen gegenüber psychischer Erkrankung, wenn eine Skala von alternativen Behandlungen angeboten wird. Auch bei den professionellen Helfern verändern sich damit grundsätzliche Überzeugungen und das klinische Vorgehen." (S. 57)

Die Rede ist hier von einer Veränderung in Italien, bei der Organisationen, die für die Pflege von Patienten mit psychischer Belastung zuständig sind, nicht mehr als Krankenhäuser, sondern primär als gemeinschaftliche Zentren gesehen werden. Konsequenterweise gibt es als Massnahme nicht mehr nur die Wahl zwischen Einweisung oder Nichteinweisung, sondern in diesen Zentren können sich ganz neue, eben differenziertere Behandlungsformen entwickeln. Patienten werden nicht mehr primär mit Medikamenten "repariert", sie werden auch nicht vor der Gesellschaft verborgen, damit sie nicht mehr stören. Die mit der Organisation verbundene Metapher ist nicht die Fabrik, die Heilung produziert, sondern eine Gemeinschaft, in der Integrationsprozesse zu einer Verbesserung der Situation der Betroffenen führen.

Ganz ähnlich ist die Frage nach dem Organisationsbild einer anderen, bemerkenswerten Institution zu stellen: dem auf Alzheimer spezialisierten Pflegehaus in Bad Blumau (Österreich). Üblicherweise werden - nicht zuletzt unter ökonomischem Druck - auch Alters- und Pflegeheime so organisiert, dass sie möglichst effizient arbeiten. Sie sind dabei oft in einer Infrastruktur gefangen, die übernommen wurde, d.h. das Management muss sich in diese Vorgaben einpassen. Treppen, Zimmerstrukturen, Aus- und Eingänge sind meist nicht neu planbar. Das Alzheimerhaus Bad Blumau hatte die einmalige Chance, das gesamte Konzept, von der Architektur über die Pflege bis zur Leistungspalette, völlig neu zu gestalten. Dies ermöglicht, dass der Patient mit seiner sehr spezifischen Krankheit in den Vordergrund gestellt wird. Alle organisatorisch-strukturellen Entscheide wurden darauf hin ausgerichtet, dass die Patienten ihre eigenständige Geschichte im Haus weiterleben können - trotz und gerade mit ihrer heimtückischen Krankheit. Die Pflegenden sind speziell ausgebildet. Die Architektur erlaubt ein freies Gehen, was ein für Alzheimer typisches Symptom unterstützt. Eine erfreuliche Nebenwirkung ist, dass weniger Medikamente benötigt werden, als dies für dieselben Patienten in einem "normalen" Pflegeheim der Fall wäre. All das wäre nicht möglich mit einem Organisationsbild, in dem eine Organisation primär die Prozesse für sich selbst effizient organisiert, oder bei dem sich die Pflegenden primär mit sich selbst befassen. 
Man könnte aber natürlich auch der Frage nachgehen, warum es in Italien überdurchschnittlich viele Familienunternehmen gibt (mögliche Antwort: weil die Organisation als Erweiterung der Familienaktivitäten gesehen wird), oder warum in den USA die Loyalität der Mitarbeitenden gegenüber der Unternehmung als tiefer angesehen wird als in anderen Ländern. Auf die Verwaltungsforschung hat sicherlich einen Einfluss, was in einem sozialen System als "gute Organisation" angesehen wird. All unsere Bilder bewirken ja auch eine normative Prägung.

Wird Organisation als ein lebendes, wertvolles System betrachtet, so wird deutlich, dass der Manager eine besondere Verantwortung übernimmt: Er muss für das Wohlergehen seiner Organisation sorgen. Er muss sie im Hier und Jetzt leistungsfähig machen, damit sie bestehen kann. Er muss sie auf die Zukunft vorbereiten, damit sie auch langfristig ihre Aufgaben wahrnehmen kann. Mit anderen Worten, er muss sich in den Dienst seiner Organisation stellen. Von besonderer Bedeutung ist diese Aussage für die öffentliche Verwaltung, aus mehreren Gründen:

a. Die öffentliche Verwaltung ist durch ihr politisches Umfeld permanent schwelenden ideologischen Konflikten ausgesetzt, die mit unklaren und kurzfristig wechselnden Zielen verbunden sind. Sie wird und muss sich daher vor Aktivismus schützen, der daraus entstehen kann.

b. Führungskräfte in der öffentlichen Verwaltung sind (noch) zu wenig sensibilisiert, dass sie eine Verantwortung für die Organisation tragen. Sie sehen sich oft eher als Ober-Sachbearbeiter denn als Manager.

c. Die übergeordnete Führungsebene, die Politiker, lassen das Bewusstsein für die Relevanz der Organisation in ihrem Zuständigkeitsbereich oft völlig vermissen. Wird Verwaltung nur als Manipulationsmasse für die eigenen egozentrischen Ziele behandelt, so verwundert es nicht, wenn sich innerhalb der Verwaltung Gegenstrategien entwickeln oder Lethargie und Demotivation breit macht.

\section{Diskussion}

Der Begriff der "managerialistischen" Verwaltungskultur spiegelt eine Veränderung des Verständnisses von Verwaltung und Verwaltungssteuerung, die nur im Kontext sinnvoll interpretiert werden kann. Das Ausmass des Managerialismus, das wahrgenommen wird, hängt massgeblich vom Betrachter und seinem eigenen Kontext ab. Einige mögliche Varianten der Kontextualisierung wären:

1. Disziplinär: Für Nicht-Betriebswirte dürften schon erste Ansätze einer stärker geführten Verwaltung nach Managerialismus aussehen, während die Betriebswirte dabei viel stärker auf die noch vorhandenen (und offensichtlichen) Defizite verweisen. Aus unserer Sicht ist das Verwaltungsmanagement in der Praxis noch längst nicht dort, wo es sein sollte, sind wir also noch weit vom Managerialismus entfernt.

2. Länderspezifisch: Im deutschsprachigen Raum lassen sich zwischen den Ländern und ihren Verwaltungskulturen grosse Unterschiede darin ausmachen, wie mit dem Thema Führung in der Verwaltung umgegangen wird. In der Schweiz werden Dinge pragmatisch umgesetzt, die in Deutschland zu grossen (strukturkonservativen) Debatten führen. Beispiele sind das Personalmanagement oder die Reform des öffentlichen Rechnungswesens. Gleichzeitig sind in Österreich zentral vorangetriebene Ent- 
wicklungen - z.B. im Electronic Government - möglich, von denen die Schweiz nur träumen kann.

3. Zeitpunktbezogen: Bestimmte Themen können reifen, können auf offene oder taube Ohren stossen, je nach dem gegenwärtigen Set an Opportunitäten. Gerade bei Reformen in der öffentlichen Verwaltung lässt sich zudem zeigen, dass die zum Zeitpunkt der Reform vorherrschende „Mode“ im Management der Privatsektors einen massgeblichen Einfluss auf den Reformansatz und die Zielvorstellung einer reformierten Verwaltung hat (Kolbe 2009: 186).

Dieser Beitrag ist ein Plädoyer für eine stärker kontextualisierte Verwaltungsforschung und lehre, als sie gerade in internationalen Publikationen erkennbar wird. Nicht ganz zu Unrecht schreibt Kuhlmann (2006):

"Ausserdem fehlt es den quantifizierenden many cases comparisons, die teils von der Prämisse 'the more (countries) the better' angeleitet scheinen, häufig in bedenklicher Weise an Kontextinformationen und landesspezifischem Kontextwissen, das aber - nicht zu verwechseln mit 'Ethnozentrismus' - unabdingbar ist, um 'Verwaltung' im jeweiligen System zu verstehen, zu deuten und letztlich auch sinnvoll zu erklären." (S. 400)

Ich habe versucht, über verschiedene Kontextschichten den Boden zu bereiten, damit ich als Forscher überhaupt in die Lage versetzt werde, die empirischen Phänomene in einer Verwaltung zu erfassen. Ich kann die "managerialistische" Verwaltung, wie sie in Neuseeland entstanden ist (und nicht etwa: entwickelt wurde), nicht losgelöst vom damaligen Kontext verstehen. Deshalb müssen alle Versuche, das neuseeländische "Modell" zu übertragen, an Unverträglichkeiten mit dem jeweiligen Kontext im Zielsystem scheitern. Das ist keine neue, keine revolutionäre Erkenntnis. Wir benötigen einen genügend komplexen Zugang zum kulturellen Kontext, der dennoch übersichtlich ist.

Als Betriebswirt, der sich seit 1991 mit öffentlicher Verwaltung befasst, halte ich den gegenwärtigen Zustand der Verwaltung in Deutschland, Österreich und der Schweiz nicht für "managerialistisch". Zwar ist unverkennbar, dass auch wir die Rhetorik des NPM übernommen haben. In verschiedenen Gemeinwesen auf allen Staatsebenen wurden Reformprojekte beschlossen, die Elemente des NPM enthalten. Damit hätten wir die ersten beiden Ebenen der Konvergenz erreicht, die Pollitt (2002) beschreibt. Sogar die Instrumentierung des NPM wurde eingekauft, selbst wenn an manchen Orten der Eindruck entsteht, dass den Anwendern noch nicht so recht klar ist, wie darauf zu spielen ist. Gleichzeitig ist aber festzuhalten, dass die Praxis in vielen Reformverwaltungen alles andere als managerialistisch ist. Dies belegen u.a. Studien aus Österreich, die zeigen, dass sich die „institutionellen Logiken“ der Verwaltung trotz NPM nur graduell, aber nicht prinzipiell verändert haben (Meyer und Hammerschmid 2006). Die alten, informellen Strukturen bestehen weiterhin, die Laufbahnmechanismen sind unverändert, und so lange die Erfolgskriterien für die Verwaltung und ihre Führungskräfte nicht auf Leistung und Wirkung ausgerichtet werden, dürfte das NPM seine volle Wirkung nie entfalten. Der Grund liegt nicht selten darin, dass eine managerialistische Verwaltung nicht in den vorherrschenden Kontext passt, und dass sich die Verwaltung deswegen dieser Art der Reform erfolgreich verweigert. 


\subsection{Konsequenzen für die Verwaltungsforschung}

Für uns Forschende lässt sich aus dem Gesagten ableiten, dass wir uns bemühen müssen, ein ganzheitliches Bild der öffentlichen Verwaltung zu erarbeiten. Wir müssen zunächst den relevanten Kontext erfassen, und zwar von aussen nach innen, nicht von innen nach aussen. Der Turm der Kontextschichten muss durchschritten werden, bevor der Forscher oder die Forscherin in die Verwaltung geht, um nach Phänomenen zu suchen, die Verwaltung zu beschreiben oder zu erklären. Der Referenzrahmen für die Forschung kann erst aus dem Verständnis des Kontextes entstehen, Verwaltungshandeln kann nur verstanden werden, wenn es in Bezug auf die besprochenen Bilderschichten interpretiert wird. Die verschiedenen Facetten des umgebenden sozialen Systems müssen angesprochen werden, der Stellenwert der Kultur in Gesellschaft und Organisation muss stärker betont werden (Schedler und Proeller 2007).

Wenn wir zu rasch in eine Verwaltung hinein gehen, um sie zu erforschen, nehmen wir wichtige Signale nicht wahr, die wir für das Verstehen dieser Verwaltung erkennen müssten. Diese Anforderung macht aber die (vergleichende) Verwaltungsforschung zu einem sehr aufwändigen Unterfangen. Noch so sophistizierte quantitative Methoden vermögen in aller Regel diesen Kontextbezug nicht herzustellen.

Als weitere Erkenntnis gilt: gute Verwaltungsforschung ist immer interdisziplinär, da der Kontext der Verwaltung hochgradig multidisziplinär ist. Es geht weniger um Ab- und Ausgrenzung von Disziplinen, als um Integration der Sichtweisen. Recht, Politik, Wirtschaft und Wissenschaft wollen prinzipiell gleichberechtigt behandelt werden. Ich hoffe, jetzt nicht die Hoffnungen meiner Kollegen aus Rechts- und Politikwissenschaft zu enttäuschen, das NPM in die Ecke des Managerialismus drängen zu können, um damit einen in seinen Extremen kategorisierten Dämon bekämpfen zu können. NPM ist nicht nur managerialistisch, sondern es ist auch wohlfahrtsstaatlich (Ruflin 2006), steuerungsbezogen (Ulrich 1994; Brühlmeier et al. 2001) und rechtsstaatlich (Mastronardi 1995; Brühlmeier et al. 1998) weiterentwickelt worden zu einem Ansatz, der längst die Züge eines Public Governance angenommen hat. Gemeinsame Forschung bedingt allerdings, dass viel Zeit für das gegenseitige Verständnis und die Wertschätzung unterschiedlicher Positionen aufgewendet wird.

Nicht ohne Grund sind im deutschsprachigen Raum Zentren der Verwaltungsforschung entstanden, die bewusst interdisziplinär arbeiten. Damit meine ich nicht nur die Anwesenheit der Disziplinen, sondern auch die aktive, konstruktive (und meist freundschaftliche) Zusammenarbeit ihrer Vertreter. Für Deutschland ist nebst Speyer in erster Linie Potsdam zu nennen, für die Schweiz Bern und St. Gallen. Ich sehe vor allem in diesen problemorientierten, interdisziplinären Ansätzen das grösste Potenzial für eine eigenständige europäische Verwaltungsforschung.

Schliesslich plädiere ich für eine Verwaltungsforschung, die auch praktisch wertschöpfend ist. Auf dem Kontinuum zwischen scientific rigor und practical relevance bewegen sich heute viele Disziplinen zu stark am methodischen Pol, d.h. sie legen grossen Wert auf methodisch einwandfreies Vorgehen, vergessen aber die Frage der praktischen Relevanz. Eine kontextualistische Forschung ist gezwungen, sich um praktische Phänomene der Verwaltung zu kümmern. Die leider zu wenigen Public Management-Lehrstühle im deutschsprachigen 
Raum, die sich mit der grössten wirtschaftlichen Branche (mit riesigem Wertschöpfungsund -vernichtungspotenzial) auseinander setzen, können es sich nicht leisten, praxisfremde Forschung zu betreiben.

\subsection{Konsequenzen für die Lehre}

Die Ausbildung junger Menschen, die sich in der Regel durch wenig Praxiserfahrung auszeichnen, muss das Bewusstsein für die Kontextabhängigkeit von sozialen Systemen schaffen. Insbesondere gilt es, die relevanten Kontextschichten der öffentlichen Verwaltung mit den Studierenden gemeinsam zu durchschreiten, anhand von Fallbeispielen die Unterschiede zwischen verschiedenen Kontextwelten zu erkennen und mögliche Konsequenzen für die Verwaltung zu diskutieren. Dies ist umso spannender, je internationaler die Zusammensetzung der Studierenden ist.

Wer Verwaltung verstehen will, der muss zwingend die für sie relevanten Disziplinen und ihre Denk- und Argumentationsweise kennen. Verwaltungsausbildung kann daher nicht rein rechtswissenschaftlich sein, sondern sie muss zugleich politik-, management- und sozialwissenschaftliche Aspekte mit einbeziehen. Immerhin bilden wir an den Universitäten und Fachhochschulen junge Menschen aus, die in einem hoch komplexen Umfeld bestehen müssen. Im Vergleich zum privatwirtschaftlichen Management ist public management um Vieles anforderungsreicher, nicht zuletzt auch in Bezug auf die Persönlichkeit.

\subsection{Konsequenzen für die Praxis}

In Gesprächen mit Praktikern stelle ich immer wieder fest, dass sie die Bedeutung der speziellen Verwaltungskultur hervor heben. Kaum ein Projekt der Verwaltungsmodernisierung kommt ohne das Ziel aus, die Kultur der Verwaltung verändern zu wollen. Solche "kulturellen Transformationsprozesse" (Hablützel 1998) können aber in einer Organisation nicht losgelöst von gesellschaftlichen Erwartungen implementiert werden. In der Praxis der Verwaltungsreform ist es daher wichtig, dass alle Beteiligten die Kontextschichten der Verwaltung analysieren und daraus entstehende Kontingenzen miteinander besprechen: was ist in diesem Kontext möglich, was nicht? Welche Botschaften lösen voraussichtlich welche Reaktionen bei den Mitgliedern der Verwaltung aus? Welche Tabus werden - bewusst oder unbewusst - angesprochen, mit welcher zu erwartenden Folge für das Veränderungsprojekt? Wie werden vermeintlich klar definierte Begriffe in diesem spezifischen Kontext interpretiert? Diese Arbeit gehört in jedem Projekt an den Anfang, in die Phase der Erwartungsklärung.

Die an der Reform Beteiligten ins Boot zu holen, heisst, ihre Sprache zu sprechen. Ich möchte diese Aussage mit einer Erfahrung belegen, die ich persönlich gemacht habe. Als wir 1994 in der Schweiz mit den ersten Konzepten für eine wirkungsorientierte Verwaltungsführung in die Öffentlichkeit gingen, waren diese auf einer stark betriebswirtschaftlichen ("managerialistischen") Grundlage entwickelt worden. Entsprechend wirtschaftlich war das Vokabular, dessen wir uns damals bedienten. Viele Verwaltungsjuristen reagierten darauf verunsichert, wenn nicht mit Unverständnis, denn Begriffe wie "Kunde", "Produkt" oder "Controlling" waren im Recht (de lege lata) nicht vorhanden. Zudem pflegen Betriebswirte bisweilen einen recht saloppen Umgang mit der Sprache und erklären Dinge gern in Grafiken, anstatt sich die Mühe zu nehmen, in eindeutiger Prosa unmissverständliche Aussagen zu machen. Diese 
(disziplinär-kulturellen) Differenzen führten zu einer Blockierung des Diskurses in der Schweiz, die erst aufgehoben wurde, als anerkannte Juristen mit einer eigentlichen "Übersetzung" des NPM ins Juristische anfingen (Mastronardi 1995; Richli 1996; Mastronardi 1999; Kettiger 2000). Der interdisziplinäre Diskurs von Mastronardi und mir ist beredtes Beispiel für diese Annäherung, die damals noch auf recht wackeligen Beinen erfolgte, und bei dem wir beide enorm dazu gelernt haben (Mastronardi und Schedler 1998, 2005). Mir will scheinen, dass diese Übersetzungsarbeit in Deutschland und in Österreich bislang zu wenig betrieben wurde, oder dass sie in der Verwaltungspraxis nicht genügend zur Kenntnis genommen wird. Aber auch dies liesse sich aus dem spezifischen Kontext erklären (was auch getan wird, vgl. beispielsweise Meyer und Hammerschmid 2006).

\subsection{Herausforderungen des Kontextualismus}

Ohne Zweifel hat auch dieser starke Kontextbezug der Verwaltungsforschung seine Grenzen. Das Studium des Kontexts ist für jeden Verwaltungsforscher zu empfehlen. Die Verwaltungswissenschaften sind jedoch gefordert, dafür Methoden zu entwickeln, die dem Versuch entgegen wirken, Erklärungen situativ oder anektotisch über subjektiv wahrgenommene "weiche" Phänomene zu suchen. Gleichzeitig ist vor allzu schneller Verallgemeinerung zu warnen. Selbst innerhalb eines kleinen Landes wie der Schweiz ist eher von Kulturen auszugehen, als von einer einheitlichen Kultur. Die Analyse der Kontextschichten ist daher - mühsam - von Fall zu Fall zu wiederholen.

Ausserdem soll Kontextforschung bestimmte Veränderungen - oder deren Verhinderung erklären, aber nicht als exogen verursacht hinnehmen. Entscheidungs- und Handlungsfreiräume erscheinen zwar zeitpunktbezogen festgelegt, sind in dynamischer Betrachtung hingegen keineswegs unveränderbar. Viele politische Diskussionen machen deutlich, welche Möglichkeiten heute bestehen, an die vor einigen Jahren niemand ernsthaft gedacht hätte. Insofern ist der Kontext ein dynamisches Element, das immer wieder neu erhoben werden muss. Verwaltungsreform hat als Wirkung, aber auch als Aufgabe, dass für die Zukunft neue Alternativen geschaffen werden können, die neue Möglichkeiten einer Verwaltungssteuerung eröffnen.

\section{Literatur}

Alford, J. und D. O'Neil, Eds. (1994). The Contract State: Public Management and the Kennett Government. Deakin Series in Public Policy and Administration. Victoria, Center for Applied Social Research, Deakin University.

Anechiarico, F. (1998). Administrative culture and civil society. Administration and Society 30(1): 13-34.

Aucoin, P. (1990). Administrative Reform in Public Management: Paradigms, Principles, Paradoxes and Pendulums. Governance: An International Journal of Policy and Administration 3(2): 115-137.

Barzelay, M. und R. Gallego (2006). From "New Institutionalism" to "Institutional Processualism": Advancing Knowledge about Public Management Policy Change. Governance 19(4): 531-557. 
Bogumil, J. (1997). Das Neue Steuerungsmodell und der Prozess der politischen Problembearbeitung - Modell ohne Realitätsbezug? Verwaltungsmodernisierung und lokale Demokratie. J. Bogumil und L. Kissler. Baden-Baden, Nomos. 2002.

Boston, J., Ed. (1995). The State under Contract. Wellington, Bridget Williams Books Ltd.

Bouckaert, G. (2007). Cultural characteristics from public management reforms worldwide. In: Cultural aspects of public management reform. K. Schedler und I. Proeller. San Diego, JAI Press: 29-64.

Brühlmeier, D., T. Haldemann, P. Mastronardi, et al. (1998). New Public Management für das Parlament: Ein Muster-Rahmenerlass WoV. Schweizerisches Zentralblatt für Staats- und Verwaltungsrecht 99(7): 297-316.

Brühlmeier, D., T. Haldemann, P. Mastronardi, et al. (2001). Politische Planung. Mittelfristige Steuerung in der wirkungsorientierten Verwaltungsführung. Bern, Stuttgart, Wien, Paul Haupt.

Brun, M., P. Mastronardi und K. Schedler (2005). Hierarchie und Netzwerke. Eine interdisziplinäre Analyse der Steuerung in der Bundesverwaltung. Bern/Stuttgart/Wien, Paul Haupt.

Brunsson, N. (2006). Mechanisms of hope. Maintaining the dream of the rational organization. Copenhagen, CBS Press.

Buschor, E. (1993). Wirkungsorientierte Verwaltungsführung - Referat an der Generalversammlung der Zürcher Handelskammer, Zürich 1. Juli 1993. Zürich, Zürcher Handelskammer.

Castles, F. G. (1994). On religion and public policy: Does Catholicism make a difference? European Journal of Political Research 25(1): 19-40.

Coleman, J. S. (1990). Foundations of social theory. Cambridge, MA, Harvard University Press.

DiMaggio, P. J. und W. W. Powell (1983). The iron cage revisited: Institutional isomorphism and collective rationality in organizational fields. American Sociological Review 48: 147160.

Fehr, E. und S. Gächter (2002). Altruistic punishment in humans. Nature 415(6868): 137-140.

Giddens, A. (1990). The consequences of modernity. Stanford, CA, Stanford University Press.

Grüning, G. (2000). Grundlagen des New Public Management. Entwicklung, theoretischer Hintergrund und wissenschaftliche Bedeutung des New Public Management aus Sicht der politisch-administrativen Wissenschaften der USA. Münster, LIT Verlag.

Hablützel, P. (1998). Verwaltungsmodernisierung als Lernprozess: Zur kulturellen Dimension des New Public Management. In: Die Ausbildung zum Public Manager. K. Schedler und C. Reichard. Bern, Stuttgart, Wien, Haupt.

Hammerschmid, G. (2001). New Public Management zwischen Konvergenz und Divergenz. Wien, Service Fachverlag.

Helmuth, U. (2009). Revising the fundamentals: insights of neuroeconomics for economic foundation of performance-related pay in public management. Transformations in Business E Economics 8(1): 21-33.

Helmuth, U. (2010). Better Performance with Performance Budgeting? Analyzing Cases of Success and Failure in Public Administrations. International Public Management Journal 13(4): 408 - 428.

Herweijer, M., Ed. (1996). 10 Jahre Tilburger Modell - Erfahrungen einer öffentlichen Verwaltung auf dem Weg zum Dienstleistungscenter. Praxis Reihe Verwaltungsreform. Bremen / Boston, SachBuchVerlag Kellner.

Hoffmann-Riem, W. (2001). Modernisierung von Recht und Justiz eine Herausforderung des Gewährleistungsstaates. Frankfurt am Main, Suhrkamp.

Hofstede, G. (1991). Cultures and organisations. Software on the mind. London, McGraw-Hill. 
Hood, C. (1991). A Public Management For All Seasons? Public Administration 1991(69): 3-19.

Hood, C. (1998). The Art of the State. Culture, Rhetoric, and Public Management. Oxford, Clarendon Press.

Jann, W. (1983). Staatliche Programme und "Verwaltungskultur". Bekämpfung des Drogenmissbrauchs und der Jugendarbeitslosigkeit in Schweden, Grossbritannien und der Bundesrepublik Deutschland im Vergleich. Opladen, Westdeutscher Verlag.

Jann, W. (1987). Strategien und Probleme der vergleichenden Verwaltungswissenschaft. In: Verwaltungsforschung in Perspektive. R. Koch. Baden-Baden, Nomos: 39-55.

Jann, W., J. Bogumil, G. Bouckaert, et al., Eds. (2004). Status-Report Verwaltungsreform. Eine Zwischenbilanz nach zehn Jahren. Modernisierung des öffentlichen Sektors. Berlin, Edition Sigma.

Jullien, F. (2005). Vortrag vor Managern über Wirksamkeit und Effizienz in China und im Westen. Berlin, Merve.

Kenis, P. und V. Schneider (1991). Policy Networks and Policy Analysis: Scrutinizing a New Analytical Toolbox. In: Policy Networks - Empirical Evidence and Theoretical Considerations. B. Marin und R. Mayntz. Frankfurt a. M., Campus Verlag: 25-62.

Kettiger, D. (2000). Wirkungsorientierte Verwaltungsführung und Gesetzgebung. Untersuchungen an der Schnittstelle zwischen New Public Management und Gesetzgebung. Bern, Stuttgart, Wien, Paul Haupt.

Kettl, D. (1993). Sharing power: public governance and private markets. Washington, D.C., Brookings.

Kettl, D. F. (1997). The global revolution in public management: driving themes, missing links. Journal of Policy Analysis and Management 16(3): 446-462.

Kolbe, P. (2009). Unde venis, homo administrans? St. Gallen, Thesis.

Kuhlmann, S. (2006). Wandel lokaler Verwaltung in Kontinentaleuropa: ein deutschfranzösischer Vergleich. In: Politik und Verwaltung. J. Bogumil, W. Jann und F. Nullmeier. Wiesbaden, Verlag für Sozialwissenschaften: 397-423.

Lapsley, I. und R. Oldfield (2001). Transforming the public sector: management consultants as agents of change. European Accounting Review 10(3): 523-543.

Mastronardi, P. (1995). Staatsrecht und Verwaltungsorganisation. Reflexionen am Beispiel des New Public Management. AJP/PJA 4(12): 1541-1553.

Mastronardi, P. (1999). Gewaltenteilung unter NPM. Schweizerisches Zentralblatt für Staatsund Verwaltungsrecht 100(9): 449-464.

Mastronardi, P. (2000). Die staatspolitische Erweiterung des NPM-Konzeptes aus rechtlicher Sicht. Verwaltung und Management 6(4): 222-227.

Mastronardi, P. und K. Schedler (1998). New Public Management in Staat und Recht - Ein Diskurs. Bern, Verlag Paul Haupt.

Mastronardi, P. und K. Schedler (2005). New Public Management in Staat und Recht. Mit einem Kommentar von Daniel Brühlmeier und einer Stellungnahme von Daniel Kettiger. Bern, Verlag Paul Haupt.

Mayntz, R. (1997). Soziologie der öffentlichen Verwaltung. Heidelberg, C.F. Müller.

Meyer, J. W. und B. Rowan (1977). Institutionalized Organization: Formal Structures as Myth and Ceremony. The American Journal of Sociology 83(2): 340-363.

Meyer, R. und G. Hammerschmid (2006). Changing institutional logics and executive identities. American Behavioural Scientist 49: 1000-1014.

Moore, M. H. (1995). Creating Public Value. Strategic Management in Government. Cambridge, MA, London, England, Harvard University Press.

Morgan, G. (2002). Bilder der Organisation. Stuttgart, Klett-Cotta. 
Mosher, L. und L. Burti (1992). Seelische Gesundheit. Ein Leitfaden für die Praxis. Bonn, Psychiatrie-Verlag.

Naschold, F., C. Reichard, W. Jann, et al., Eds. (1996). Leistungstiefe im öffentlichen Sektor. Erfahrungen, Konzepte, Methoden. Modernisierung des öffentlichen Sektors. Berlin, Sigma.

Osborne, D. und T. Gaebler (1992). Reinventing Government: How the entrepreneurial Spirit is transforming the public Sector. Reading, Addison-Wesley Publishing Company, Inc.

Pierson, P. (2000). Increasing Returns, Path Dependence, and the Study of Politics. The American Political Science Review 94(2): 251-267.

Pollitt, C. (1990). Managerialism and the Public Service: An Anglo-American Experience. Oxford, Blackwell.

Pollitt, C. (2002). Clarifying convergence: Striking similarities and durable differences in public management reform. Public Management Review 4(1): 471-492.

Pollitt, C. und G. Bouckaert (2000). Public Management Reform: a Comparative Analysis. Oxford, Oxford University Press.

Proeller, I. und K. Schedler (2005). Change and continuity in the continental tradition of public management. In: The Oxford Handbook of Public Management. E. Ferlie, L. E. Lynn und C. Pollitt. Oxford, Oxford University Press: 695-719.

Reichard, C. (1994). Umdenken im Rathaus - Neue Steuerungsmodelle in der deutschen Kommunalverwaltung. Berlin, Edition Sigma.

Richli, P. (1996). Öffentliches Dienstrecht im Zeichen des New Public Management staatsrechtliche Fixpunkte für die Flexibilisierung und Dynamisierung des Beamtenverhältnisses. Bern, Stämpfli.

Rüegg-Stürm, J. (2002). Das neue St. Galler Management Modell. Bern/Stuttgart/Wien, Paul Haupt.

Ruflin, R. (2006). Wohlfahrtsstaatliches Kontraktmanagement : die Verhandlung und Umsetzung von Leistungsverträgen als Herausforderung für Nonprofit-Organisationen. Bern/Stuttgart/Wien, Paul Haupt.

Schedler, K. (1995). Ansätze einer wirkungsorientierten Verwaltungsführung. Von der Idee des New Public Management (NPM) zum konkreten Gestaltungsmodell. Fallbeispiel Schweiz. Bern/Stuttgart/Wien, Haupt.

Schedler, K. (2008). The study of public management in Switzerland. In: The study of public management in Europe and the US. W. Kickert. London, Routledge: 144-166.

Schedler, K. und J. Felix (2000). Quality in public management: the customer perspective. International Public Management Journal Nr. 3: 125-143.

Schedler, K. und I. Proeller (2000). New Public Management. Bern, Haupt.

Schedler, K. und I. Proeller, Eds. (2007). Cultural aspects of public management reform. Oxford, Elsevier.

Schein, E. H. (1992). Organizational culture and leadership. San Francisco, Jossey-Bass.

Schindler, B. (2010). Verwaltungsermessen: Gestaltungskompetenzen der öffentlichen Verwaltung in der Schweiz. Baden-Baden, Nomos.

Thompson, F. und L. R. Jones (1986). Controllership in the Public Sector. Journal of Policy Analysis and Management 5(3).

Ulrich, G. (1994). Politische Steuerung - Staatliche Intervention aus systemtheoretischer Sicht. Opladen, Leske + Budrich.

Von Maravic, P. und C. Reichard (2003). New Public Management and Corruption: IPMN Dialogue and Analysis. International Public Management Review 4(1): 84-130.

Weber, M. (1947). Wirtschaft und Gesellschaft. Tübingen, Mohr (Siebeck). 
Weick, K. E. (1995). Sensemaking in organizations. Thousand Oaks, Sage.

Wise, L. R. (2002). Public Management Reform: Competing Drivers of Change Public Administration Review 62(5): 556-567.

Wunderer, R. (1997). Führung und Zusammenarbeit: Beiträge zu einer unternehmerischen Führungslehre. Stuttgart, Schäffer-Poeschel.

Zauner, A., P. Heimerl-Wagner, W. Mayrhofer, et al. (2006). Von der Subvention zum Leistungsvertrag. Neue Koordinations- und Steuerungsformen und ihre Konsequenzen für Nonprofit-Organisationen - eine systemtheoretische Analyse. Bern, Haupt. 